\title{
Solid-cystic papillary tumour of the pancreas
}

\author{
B.D. Dhaigude, MBBS, MS (General Surgery) ${ }_{2}^{1}$ \\ R.N. Bhardwaj, MBBS, MS (General Surgery) ${ }^{2}$ \\ Manan Singh, MBBS, MS(General Surgery, Resident) ${ }^{3}$ \\ Shreyas Bhadranavar, MBBS, MS (General Surgery, Resident) 4 \\ Abhishek Kunal, MBBS MS (General Surgery, Resident) ${ }^{5}$ \\ Vishwanath Muppa, MBBS MS (General Surgery, Resident) ${ }^{6}$
}

1-6 Department Of General Surgery, Pad. Dr. DY Patil Medical College, Sant Tukaram nagar, Pimpri, Pune, Maharashtra, India.

Key words: Solid - cystic papillary tumour of pancreas; Distal pancreatectomy.

\section{Introduction}

Solid-cystic papillary tumours of pancreas (SCPTs) are rare. This tumour mainly occurs in women in the second to fourth decade of life. Franz first described this tumour in 1959 [1] as "papillary tumour of pancreas, benign or malignant [1]. Though rare, the incidence of this tumour has been increasing in recent years. Surgical resection is associated with an excellent prognosis. However, the main preoperative problem encountered is misdiagnosis of the tumour on imaging.

\section{Case summary}

A 28 year old woman presented to our institute with vague abdominal pain for two months. The obstetric history, presented childbirth four months back. No history of vomiting, weight loss, altered bowel/bladder habits. Abdominal examination revealed a mass which was involving left hypochondriac and lumbar region which was cystic in consistency, mobile and tender and was not crossing midline. All laboratory parameters including serum amylase, Carcinoembryogenic antigen (CEA), alpha- fetoprotein (AFP) and carbohydrate antigen (CA) 19-9 were all in normal range. Ultrasound of abdomen revealed a mass about $9.0 \mathrm{~cm} \times 7.1 \mathrm{~cm} \times 7.0 \mathrm{~cm}$ diameter in left lumbar region, probably located in transverse mesocolon close to splenic flexure and mesenteric mass as second possibility. The mass is not retroperitoneal and has $3 \mathrm{~mm}$ thick uniform wall with smooth surface. Triple phase MDCT scan of abdomen revealed (figure 1), a well defined rounded heterogenous density lesion, capsulated, measuring $8.5 \mathrm{~cm} \mathrm{x}$ $9 \mathrm{~cm} \times 9 \mathrm{~cm}$, seen in the pancreatic tail region involving the adjacent mesentery. Central hyperdense and non- enhanc-

Correspondence: Dr. Manan Singh

E-Mail: mananchd@gmail.com

Phone Number. +91 9545714448

Address: Dept. Of General Surgery, Pad. Dr DY Patil

Medical College, Sant Tukaram nagar, Pimpri, Pune,

Maharashtra , India. PIN code 411018 ing hypodense components are seen within it. The rest of pancreas shows normal appearance. The splenic vein is compressed and displaced posteriorly. The lesion is also indenting greater curvature of the stomach and displacing the splenic flexure of the colon inferiorly. The findings are likely to represent a rather benign looking lesion? Mesenteric desmoid tumor? papillary cyst adenoma pancreas?

The patient was taken to exploratory laparotomy. Intraoperatively it was found that, a cystic mass about $10 \mathrm{~cm} \times$ $9 \mathrm{~cm}$ adherent to transverse mesocolon, displacing splenic vessels superoposteriorly and indenting posterior wall of stomach, arising from tail of pancreas (figure 2). There was no invasion or infiltration of surrounding structures, vessels and para-aortic, pre-aortic lymphadenopathy. Distal pancreatectomy without splenectomy done. histopathological revealed solid cystic papillary tumour of pancreas. Postoperative period was uneventful and patient is disease free at follow up of six months.

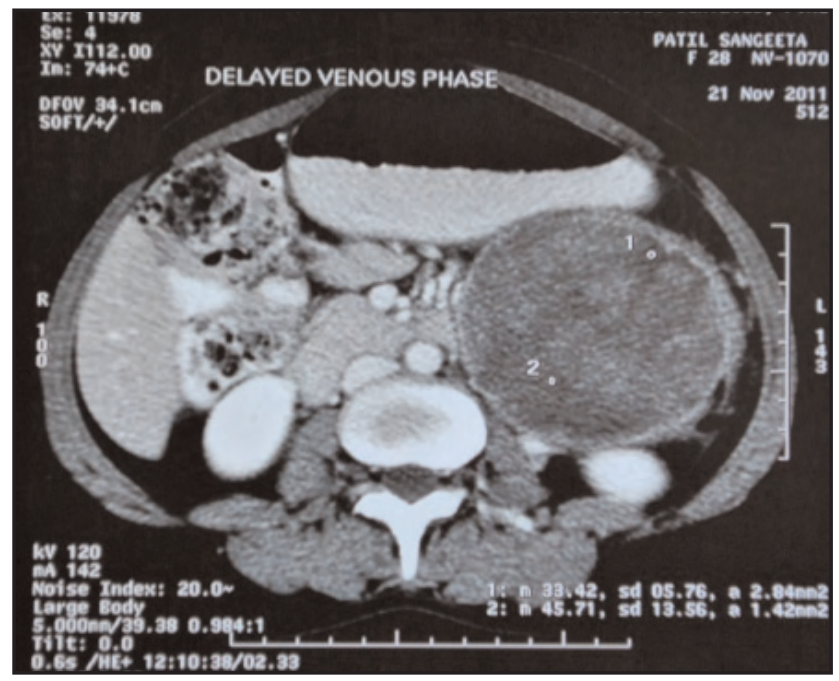

Figure 1: Computerised tomogram of the abdomen showing the cystic tumour in the tail of the pancreas 


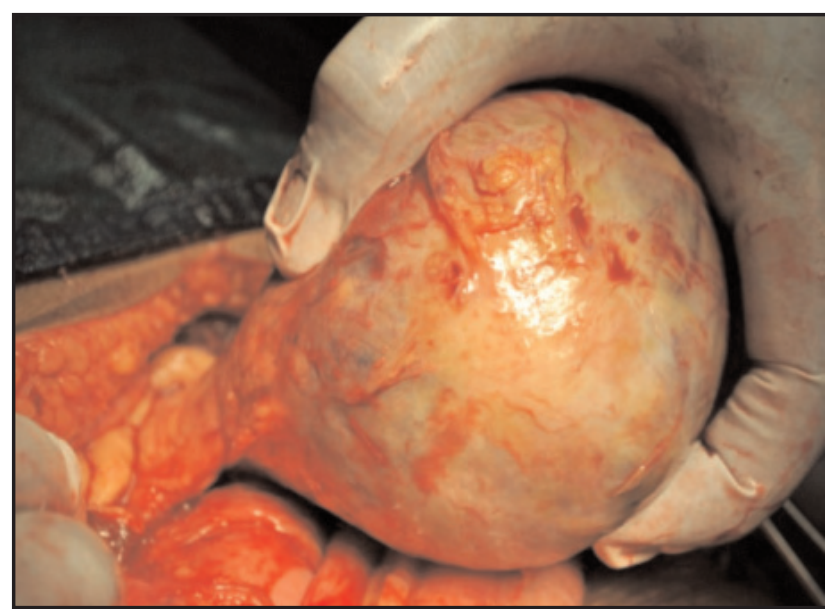

Figure 2: Operative photograph of the tail of pancreas cystic tumour

\section{Discussion}

SCPTs commonly arises in the tail and the body of pancreas and very rarely involves the head [2] and represent about $2 \%$ of all pancreatic tumours and $9.3 \%$ of cystic pancreatic neoplasms.

This pancreatic cystic tumour which develops exclusively in women, commonly before 40 years of age and most frequently has a benign clinical progression with rare metastasis and low tendency to extend locally [3], female hormones play an important role in the growth but not in the manifestation of SPT [4]. Pregnancy gives a powerful augmentative stimulus through the secretion of progesterones.

The origin and histogenesis of SCPTs is controversial, with the tumour expressing epithelial as well as mesenchymal markers [2]. Mitosis is quite uncommon and this confirms the low grade of malignancy.

CT (Computed Tomography) scan, Ultrasonography (US) and Endosonography (EUS) have been used with variable success in diagnosing solid cystic pancreatic tumor. CT scan and EUS are more sensitive and specific and have shown more accuracy in diagnosing SCPTs. Typically a large, well-defined, encapsulated lesion with heterogeneous high or low signal intensity on $\mathrm{T} 1$ and T-2-weighted, is found on gadolinium-enhanced dynamic MRI. In our case we relied on CT scan report.

Depending on the location of SCPTs, the surgical operation is chosen.

\section{Conclusion}

In conclusion, a diagnosis of SCPTs should be considered in young women presenting with a large, round, welldefined pancreatic mass. Solid cystic papillary tumors of the pancreas should be treated surgically, pancreatic resections in localised tumors, and aggressive treatment with complete resection of both the primary tumor and metastatic lesions with postoperative radiotherapy is recommended. Surgical excision offers the best chance for cure and should always be attempted irrespective of the magnitude of resection involved. Patients with solid cystic papillary tumour of pancreas have an excellent prognosis after surgical excision.

\section{References}

1. Frantz VK. Tumors of the pancreas. In: Atlas of Tumor Pathology, Section 7, Fascicles 27 and 28. Washington, DC, USA: Armed Forces Institute of Pathology, 1959;32-3.

2. Lee WJ, Park YT, Choi JS, Chi HS, Kim BR. Solid and papillary neoplasm of the pancreas. Yonsei Med J 1996; 37:131-41.

3. Fulvio Freda, Eugenio Procaccini, Roberto Ruggerio, Massimo Antropoli, Amelia Manganiello, Luigi nunziata, Pasquale Petronella and Francesco Lo Schiavo. Solid-cystic pseudopapillary tumour of pancreas: description of two cases and literature review. Tumori 2007;93:522-525

4. Morales A, Ruiz Molina JM, Esteves HO, Robles-Diaz G, Diaz- Sanchez V: Papillary-cystic neoplasm of the pancreas. A sexsteroid dependent tumor. Int J Pancreatol, 1998; 24: 219225

\begin{tabular}{|l|l|}
\hline LOCATION & TYPE OF OPERATION \\
\hline Head & Pylorus-preserving pancreaticoduodenectomy \\
\hline Neck and body & $\begin{array}{l}\text { resection by central pancreatectomy and re-implanta } \\
\text { tion of the pancreatic remnant into the stomach }\end{array}$ \\
\hline Pancreatic tail or body of pancreas & Distal pancreatectomy with splenectomy \\
\hline
\end{tabular}

